\title{
Ophthalmological Considerations for COVID-19 Vaccination in Patients with Inflammatory Eye Diseases and Autoimmune Disorders
}

\author{
Charlene Y. C. Chau • Loraine L. W. Chow - Siddharth Sridhar • \\ Kendrick C. Shih (1)
}

Received: January 15, 2021 / Accepted: February 16, 2021 / Published online: March 6, 2021

(c) The Author(s) 2021

\section{ABSTRACT}

The global impact imposed by the coronavirus disease 2019 (COVID-19) pandemic may be soon alleviated by the introduction and worldwide dissemination of safe and effective vaccines. This expedited timetable for development and approval of COVID-19 vaccines is an unprecedented extraordinary, concerted achievement by the scientific community. With the pending global rollout of vaccines, each with different mechanisms of action, physicians of various specialties will need to identify vulnerable patient groups for special considerations or advice. In this commentary, we analyse the important considerations for COVID-19 vaccines in patients with inflammatory eye diseases. Scrutiny of immunogenicity and adverse effects, particularly antibody-dependent enhancement, would better help in counselling these patients undergoing vaccination. More research on pharmacovigilance would

C. Y. C. Chau - L. L. W. Chow · K. C. Shih $(\bowtie)$ Department of Ophthalmology, Li Ka Shing Faculty of Medicine, The University of Hong Kong, Pok Fu Lam, Hong Kong SAR

e-mail: kcshih@hku.hk

\section{S. Sridhar}

Department of Microbiology, Li Ka Shing Faculty of Medicine, The University of Hong Kong, Pok Fu Lam, Hong Kong SAR allow for tailored guidelines and personalised management strategies.

Keywords: Autoimmune disorders; COVID-19; Inflammatory eye disease; Ophthalmic considerations; Pandemic; Vaccination

\section{Key Summary Points}

As production and distribution of the COVID-19 vaccines gathers momentum, physicians will need to be aware of special considerations for vulnerable patients, such as those with inflammatory eye diseases.

There is also potential for lower vaccine efficacy in pharmacologically immunosuppressed patients.

Furthermore, physicians and ophthalmologists may need to be aware of the possibility of vaccine-induced antibody-dependent enhancement of preexisting inflammatory eye diseases.

Above all, it is important to emphasize the need to maintain preventive measures in protecting oneself against COVID-19 infection while the pandemic continues, even after vaccination. 


\section{DIGITAL FEATURES}

This article is published with digital features, including a summary slide to facilitate understanding of the article. To view digital features for this article go to https://doi.org/10.6084/ m9.figshare.14040062.

\section{INTRODUCTION}

The worldwide emergence of the severe acute coronavirus syndrome coronavirus 2 (SARSCoV-2) has, to date, affected more than 86 million people globally since the World Health Organization (WHO)'s pandemic declaration on 11 March $2020[1,2]$. The deleterious health consequences and socioeconomic reverberations of the coronavirus disease 2019 (COVID-19) have made accelerated development of therapeutics and vaccines exigent. Expedited development of vaccines was availed by the Access to COVID-19 Tools Accelerator (ACT-A) - a global collaboration amongst organisations such as the WHO, GAVI, and the Coalition for Epidemic Preparedness Innovations (CEPI). As of December 2020, there are 71 vaccine candidates (23 in phase I, 32 in phase II, 18 in phase III) in the clinical development process [3]. Currently, nine vaccinesBNT162b2 (BioNTech/Pfizer), Sputnik V (Gamelaya), mRNA-1273 (Moderna), ChAdOx1 (University of Oxford), BBIBP-CorV (Sinopharm), Covishield (Serum Institute of India), Covaxin (Bharat Biotech), CoronaVac (Sinovaac), and Ad5-nCoV (Cansino Biologics)- have been approved for use $[3,4]$. The last four are approved for early or limited use in a few countries.

There is limited data to guide the use of COVID-19 vaccines for patients with underlying ophthalmological diseases. This may be attributable to the unprecedented time frame of vaccine development and approval. Vaccine development typically spans across $10-15$ years from initial scientific discovery, preclinical and clinical studies, to licensure [5]. The sped-up timeline may be attributable to the rapid deployment of flexible vaccine platforms, generous funding, innovative trial designs and multinational cooperation. Timely legislative changes by pharmaceutical regulatory bodies have also contributed to the streamlined vaccine development process [6].

Traditionally, clinical trials consist of four successive phases:

(1) Phase I assesses the safety profile and immunogenicity of vaccine candidates by testing in a small group of healthy individuals $(<100)$.

(2) Phase II studies the safety and immunogenicity of the vaccine in a larger group of a few hundred individuals. These individuals would include at-risk populations. The optimal dose, vaccine regimen and method of delivery are also assessed.

(3) Phase III further evaluates the efficacy and safety of the vaccine in a larger population of people in different regions and countries (in thousands).

(4) Phase IV involves safety surveillance after the drug has been approved for use.

This pandemic necessitated a new trial design. Rather than implementing phases in succession, phases may overlap or be combined. Phase III trials are swiftly commenced upon interim analysis of phase I/II trials, whilst commercial production may begin while awaiting results from phase III [7]. The homogenous study populations in pre-marketing randomised clinical trials preclude the assessment of vaccine usage in patients with underlying ophthalmological pathologies. The lack of pharmacovigilance studies as of yet restricts vaccination guidelines for special subpopulations to only a hypothetical basis. Herein, we propose several key considerations for physicians when considering administration of COVID-19 vaccines to patients with ophthalmological diseases. This article is based on previously conducted studies and does not contain any new studies with human participants or animals performed by any of the authors.

\section{Immunogenicity of the Vaccine}

The immunological basis of vaccines lies in the priming of the antiviral innate immune response followed by the activation of adaptive 
immunity for long-term immunological memory. Briefly, microbial components contained within vaccines or expressed by vaccines in human cells are recognised by antigen-presenting cells (APCs), triggering downstream signalling cascades and production of cytokines and chemokines [8]. Migration of APCs to draining lymph nodes and the subsequent sensitisation of T cells activate antigen-specific cellmediated and humoral responses to varying degrees depending on the type of vaccine [9]. A small subset of $\mathrm{T}$ and $\mathrm{B}$ cells that was clonally expanded during the course of primary immune response survives as memory cells, which allows for a secondary response that is of higher speed and magnitude upon re-encounter with the same pathogen [10]. The immunogenicity of the candidate vaccine, i.e. the ability to generate an immune response, constitutes an important endpoint for phase I and II clinical trials. For COVID-19 trials, the immunogenicity profile is evaluated on the basis of the titre of neutralising antibodies, although many studies have also evaluated $\mathrm{T}$ cell responses as an equally important correlate of protection [11]. Similar to other vaccines, the immune response shows high interpersonal heterogeneity, with immunosenescence or immunosuppression being important factors driving variation [12]. These demographics are commonly encountered in ophthalmology. For instance, the immunological underpinnings of various eye diseases may reflect a derangement in systemic immunity despite the eye being a prototypic immune-privileged organ [13]. Another example would be the ocular effects of autoimmune disorders; the altered immunological state may stem from the original disease or a consequence of immunosuppressive therapy use. Therefore, more data for efficacy and safety is key to inform the use of vaccines in these demographic populations [14].

Patients who are pharmacologically immunosuppressed constituted a significant group of vulnerable patients for ophthalmologists during the pandemic. Immunosuppressive therapy is increasingly used to manage ocular inflammation, which is a major cause of ocular morbidity. The dilemma over the use, timing, dosage and duration of immunosuppression in patients with COVID-19 persists [15]. The conundrum will likely extend to COVID-19 vaccine administration given the limited safety and efficacy data. A risk-benefit analysis may be challenging. First, the relationship between impaired immune status and disease outcomes is unclear. Immunosuppressed individuals are, by default, at increased risk of infections. Yet, anecdotal evidence points to an asymptomatic or mild course of disease in immunocompromised patients, such as organ transplant recipients and patients with HIV [16-18]. The immunosuppression is likely beneficial in dampening the hyperinflammation, which is the major driver of organ damage [19]. On the other hand, impairment of antimicrobial immunity may increase the viral load and drives inflammation, thus severity [20]. This could also be explained by the occurrence of locus minoris resistentiae with the eye being an immunologically privileged organ. Second, the weakened immune systems may undermine the immunogenicity of the vaccine. This has been demonstrated in other vaccines, with lower seroprotection and seroconversion rates observed when compared with healthy controls [21]. However, immunogenicity may not always correlate with clinical efficacy [22]. Nevertheless, it would be important to remind such patients, even after vaccination, to continue measures to protect themselves from COVID-19 infection during the pandemic. Third, the safety profile has not been assessed in this group of patients. Ocular inflammatory diseases can occur in isolation or as part of systemic autoimmune diseases, such as systemic lupus erythematosus (SLE) and Sjögren's syndrome. The immunogenicity of COVID-19 vaccines could potentially induce or trigger autoimmune diseases. Autoimmunity may be stimulated antigen-specifically, i.e. via molecular mimicry between microbial antigen and that of the host, or non-antigen-specifically, i.e. secondary to heightened innate immune response or the release of sequestered self-antigens [23]. Associations between autoimmunity and vaccines, albeit rare, have been reported [24]. Maillefert et al. reported development, exacerbation or relapse of rheumatic disorders following hepatitis B vaccination, but a causal relationship 
could not be established [25]. Contrastingly, a prospective study on 27 patients with SLE showed no statistically significant difference in flare rates between vaccinated and non-vaccinated subjects [26]. It is important to note that the presence of a flare is assessed by an index that considers organ manifestations, which may not take into consideration other parameters of flare such as hospitalisations and changes to medications [27]. Additional questions should also be addressed: (1) Should immunosuppressive or immunomodulatory treatments be altered? Reduced dosage or a bridging therapy with local and regional therapies have already been observed in patients with active uveitis since the pandemic $[28,29]$. However, the risk of disease relapse may have grave consequences on visual function and quality of life. Whilst this approach was considered to minimise the susceptibility to severe respiratory infections, whether this should be considered in the context of immunisation against SARS-CoV2 requires further consideration. (2) What is the optimal timing of vaccination, in relation to disease activity and the timing of immunosuppressant usage? Most vaccination studies conducted in patients with autoimmune inflammatory rheumatic diseases include patients at the quiescent stage, with insufficient data for those with moderate to severe disease activity [22]. The recommendations from the European League Against Rheumatism (EULAR) favour vaccination during quiescent disease and preferably before initiating immunosuppression if without indications for urgent administration [22]. However, this should be considered on a case-by-case basis with shared decision-making, taking into account patients' preference, concerns, and risk of acquiring COVID-19.

\section{Adverse Events of COVID-19 Vaccines}

A meta-analysis of five randomised, doubleblind, placebo-controlled trials of COVID-19 vaccine candidates noted that local and systemic adverse events reported were all transient, and mostly mild or moderate [30]. Common local adverse reactions include pain, itching, redness, swelling and induration. Systemic reactions, such as fatigue, headache, muscle pain, joint pain and malaise/anorexia are significantly more likely in vaccinated subjects compared to control [30]. However, the spectrum of adverse effects may not yet be fully demonstrated given the relatively small number of studies included and the exclusion of singleblind or placebo-free studies. Notably, some approved vaccine candidates such as the ChAdOx1 nCoV-19 were excluded from the analysis.

Antibody-dependent enhancement (ADE) is a concern for the development of COVID-19 vaccines. Whilst high-affinity antibodies against epitopes of viral particles may result in elimination of the virus, heterotypic (non-neutralising) antibodies or of suboptimal concentration will worsen disease severity. Two distinct mechanisms in which antibodies are exploited to enhance viral infection have been documented. Enhanced virus uptake occurs when the $\mathrm{Fc}$ region of the antibody-virus immunocomplex binds to $\mathrm{Fc} \gamma$ receptor $(\mathrm{Fc} \gamma \mathrm{R})$ expressing phagocytic cells. This may lead to an increase in virus-infected cells (termed extrinsic $\mathrm{ADE}$ ), or heightened viral replication with modulation of the antiviral signalling pathway (termed intrinsic ADE) [31]. ADE has been documented in preclinical studies of vaccine candidates against severe acute respiratory syndrome (SARS) and the Middle East respiratory syndrome (MERS) [32]. It is theorised that the risk of ADE is higher in inactivated vaccines. Inactivated vaccines are promising given their speed and ease of production, safety profile compared to live attenuated vaccines, and high potency [33, 34]. However, the presentation of multiple epitopes, particularly at immunodominant sites in close proximity to the receptor binding domain of the spike protein, may precipitate the production of sub- or nonneutralising antibodies $[35,36]$. The subsequent virus internalisation will exacerbate pre-existing infection or induce harmful immunopathology. There is inconclusive evidence to suggest the occurrence of ADE in SARS-CoV2 infections. It is encouraging that $\mathrm{ADE}$ was not observed in preclinical studies of the approved inactivated vaccines, BBIBP-CorV and CoronaVac, in rhesus 
macaques [37, 38]. CoronaVac's 100\% efficacy against severe COVID-19 further suggests a low risk of ADE. Yet, larger-scale phase III trials will provide critical data regarding its safety profile. Human convalescent plasma trials have also not shown definite signals of ADE [39-41]. The impact of immunological memory to endemic coronaviruses on SARS-CoV2 acquisition and clinical outcomes remains unclear. The crossreactive antibodies to endemic coronaviruses, which have extensive sequence homology to SARS-CoV2, may mediate ADE without viral neutralisation [42]. Substantial cross-reactivity to spike glycoproteins, which is essential for viral entry and fusion, has been observed in plasma in infected patients $[43,44]$. Yet a retrospective study analysing the comprehensive respiratory panel PCR (CRP-PCR) data in 15,928 patients suggests that prior coronavirus exposure may be protective against severe COVID-19 [45]. It is important to note that the retrospective nature of the study, the small sample size and the differences in assays may introduce bias.

In the context of COVID-19, ADE may precipitate ocular or neuro-ophthalmological manifestations, with the former shown to have a positive correlation with increased disease severity [46].

\section{Ocular Manifestations: Impacts on Anterior Segment}

(1) Conjunctivitis. Unilateral and bilateral conjunctivitis have been reported [47-51]. Signs and symptoms may range from mild (e.g. tearing, conjunctival hyperaemia, foreign body sensation) to severe (e.g. photophobia, chemosis, follicular reaction) $[46,52]$. The timing of presentation is variable; conjunctivitis may appear as the sole presenting sign, an initial symptom, synchronously with other well-known constitutional and respiratory symptoms, or in the late stage after serological clearance [50, 51, 53, 54]. Whilst mostly resolved within 1-2 weeks without complications, relapse has been reported in a case report [52, 55].

(2) Keratitis and episcleritis. Albeit rare, superficial punctate keratitis and keratoconjunctivitis have been described in case studies $[48,56]$. Episcleral involvement has also been documented $[57,58]$.

\section{Ocular Manifestations: Impacts on Posterior Segment}

The effect of COVID-19 on the retina and the optic nerve is only reported in small case studies. The virus may affect ganglion cells and inner plexiform layers, optic discs and retina [59-61].

\section{Neuro-Ophthalmological Manifestations}

Ophthalmoplegia secondary to cranial nerve palsies has been reported $[62,63]$. Possible postulated explanations include direct viral invasion, post-viral acute demyelinating inflammatory polyneuropathy, and ischaemia.

There remain several questions to be elucidated. The first pertains to the paradoxical nature of the protective effects versus ADE risk of vaccines. Granted the uncertainty over the relevance of ADE in patients with COVID-19, would the administration of vaccine imply the protection from SARS-CoV-2, thus in turn associated ocular and neuro-ophthalmological pathologies? Second, do chronic eye diseases confer additional risk to ADE-mediated ophthalmological complications? This has not been discussed in the extant literature. However, an analogous comparison may be the relationship between chronic eye diseases and neuro-ophthalmological presentation of SARS-CoV-2. The ocular manifestations of COVID-19 could be explained by the ocular tropism of respiratory viruses due to the anatomical and cellular similarities between ocular and respiratory systems [64]. Therefore, it may be inferred that eye diseases with altered anatomical or mucosal immune properties may affect susceptibility to virus-related ocular diseases [65]. Other pathogeneses may account for the ophthalmological symptoms, for instance immune-vascular inflammation and thrombotic complications $[57,60]$. A prospective cohort study of ocular manifestations of COVID-19 did not demonstrate a statistically significant increase in the risk of conjunctival congestion in patients with chronic eye diseases (e.g. conjunctivitis, 
keratitis, xerophthalmia) [54]. Third, the implications of ADE for ophthalmological patients may not be straightforward. The transient nature of ocular manifestations, such as conjunctivitis, may not warrant vaccines as a contraindication to patients with underlying anterior segment disorders. That said, the management approach needs to be considered should these ocular complications arise. Whilst dependent on the severity of the presentation, guidelines as to when a conservative approach or an escalated treatment strategy should be implemented would be ideal.

\section{CONCLUSION}

The global rollout of the COVID-19 vaccines is a crucial step in controlling the current pandemic. As proven in phase III clinical trials, the leading vaccines under production are safe and effective in protecting patients from symptoms and severe illness due to COVID-19. As production and distribution of the vaccines gathers momentum, medical professionals will need to be aware of special considerations for vulnerable patient groups under their care, such as patients with inflammatory eye diseases and autoimmune disorders. While long-term evidence from clinical trials is still lacking, this commentary highlights several key areas that should be of importance during counselling of patients with underlying inflammatory eye diseases, with or without immunosuppressive treatment. This includes the potential for lower vaccine efficacy in pharmacologically immunosuppressed patients and the potential risk of vaccine-induced ophthalmic ADEs. Above all, it is important to emphasize the need to maintain preventive measures in protecting oneself against COVID-19 infection while the pandemic continues, even after vaccination.

\section{ACKNOWLEDGEMENTS}

Funding. No funding or sponsorship was received for this study or publication of this article. The Rapid Service Fee was funded by the authors.

Authorship. All named authors meet the International Committee of Medical Journal Editors (ICMJE) criteria for authorship for this article, take responsibility for the integrity of the work as a whole, and have given their approval for this version to be published.

Author Contributions. Charlene YC Chau, Kendrick C Shih, Loraine LW Chow, Siddharth Sridhar were involved in study design, data collection, data analysis, manuscript writing and editing.

Disclosures. Charlene YC Chau, Kendrick C Shih, Loraine LW Chow, Siddharth Sridhar have nothing to disclose.

Compliance with Ethics Guidelines. This article is based on previously conducted studies and does not contain any new studies with human participants or animals performed by any of the authors.

Data Availability. The authors agree to make all materials, data and associated protocols promptly available to readers without undue qualifications in material transfer agreements.

Open Access. This article is licensed under a Creative Commons Attribution-NonCommercial 4.0 International License, which permits any non-commercial use, sharing, adaptation, distribution and reproduction in any medium or format, as long as you give appropriate credit to the original author(s) and the source, provide a link to the Creative Commons licence, and indicate if changes were made. The images or other third party material in this article are included in the article's Creative Commons licence, unless indicated otherwise in a credit line to the material. If material is not included in the article's Creative Commons licence and your intended use is not permitted by statutory regulation or exceeds the permitted use, you will need to obtain permission directly from the copyright holder. To view a copy of this licence, 
visit http://creativecommons.org/licenses/bync/4.0/.

\section{REFERENCES}

1. Dong E, Du H, Gardner L. An interactive web-based dashboard to track COVID-19 in real time. Lancet Infect Dis. 2020;20(5):533-4.

2. World Health Organization. WHO Director-General's opening remarks at the media briefing on COVID-19, 11 March 2020. https://www.who.int/ director-general/speeches/detail/who-directorgeneral-s-opening-remarks-at-the-media-briefingon-covid-19-11-march-2020. Accessed 31 Dec 2020.

3. McGill COVID19 Vaccine Tracker Team. 2020. https://covid19.trackvaccines.org/vaccines/. Accessed 31 Dec 2020.

4. Zhao J, Zhao S, Ou J, et al. COVID-19: coronavirus vaccine development updates. Front Immunol. 2020;11:602256-602256.

5. Petousis-Harris H. Assessing the safety of COVID-19 vaccines: a primer. Drug Saf. 2020;43(12):1205-10.

6. Van Norman GA. Update to drugs, devices, and the FDA: how recent legislative changes have impacted approval of new therapies. JACC Basic Transl Sci. 2020;5(8):831-9.

7. Krammer F. SARS-CoV-2 vaccines in development. Nature. 2020;586(7830):516-27.

8. Kang SM, Compans RW. Host responses from innate to adaptive immunity after vaccination: molecular and cellular events. Mol Cells. 2009;27(1):5-14.

9. Janeway CATP, Walport $\mathrm{M}$, et al. Principles of innate and adaptive immunity, in immunobiology: the immune system in health and disease. New York: Garland Science; 2001.

10. Sallusto F, Lanzavecchia A, Araki K, Ahmed R. From vaccines to memory and back. Immunity. 2010;33(4):451-63.

11. Dong Y, Dai T, Wei Y, Zhang L, Zheng M, Zhou F. A systematic review of SARS-CoV-2 vaccine candidates. Signal Transduct Target Ther. 2020;5(1):237.

12. Walsh EE, Frenck RW Jr, Falsey AR, et al. Safety and immunogenicity of two RNA-based Covid-19 vaccine candidates. N Engl J Med. 2020;383(25): 2439-50.
13. Perez VL, Caspi RR. Immune mechanisms in inflammatory and degenerative eye disease. Trends Immunol. 2015;36(6):354-63.

14. DeFrancesco L. Whither COVID-19 vaccines? Nat Biotechnol. 2020;38(10):1132-45.

15. Thng ZX, De Smet MD, Lee CS, et al. COVID-19 and immunosuppression: a review of current clinical experiences and implications for ophthalmology patients taking immunosuppressive drugs. $\mathrm{Br} \mathrm{J}$ Ophthalmol. 2020;2020:bjophthalmol-2020316586.

16. Bussalino E, De Maria A, Russo R, Paoletti E. Immunosuppressive therapy maintenance in a kidney transplant recipient with SARS-CoV-2 pneumonia: a case report. Am J Transplant. 2020;20(7):1922-4.

17. D'Antiga L. Coronaviruses and immunosuppressed patients: the facts during the third epidemic. Liver Transpl. 2020;26(6):832-4.

18. Blanco JL, Ambrosioni J, Garcia F, et al. COVID-19 in patients with HIV: clinical case series. Lancet HIV. 2020;7(5):e314-6.

19. Martins-Chaves RR, Gomes CC, Gomez RS. Immunocompromised patients and coronavirus disease 2019: a review and recommendations for dental health care. Brazil Oral Res. 2020;2020:34.

20. Ritchie AI, Singanayagam A. Immunosuppression for hyperinflammation in COVID-19: a doubleedged sword? Lancet. 2020;395(10230):1111-1111.

21. Liang Y, Meng FY, Pan HF, Ye DQ. A literature review on the patients with autoimmune diseases following vaccination against infections. Hum Vaccin Immunother. 2015;11(9):2274-80.

22. Furer V, Rondaan C, Heijstek MW, et al. 2019 update of EULAR recommendations for vaccination in adult patients with autoimmune inflammatory rheumatic diseases. Ann Rheum Dis. 2020;79(1):39.

23. Wraith DC, Goldman M, Lambert P-H. Vaccination and autoimmune disease: what is the evidence? Lancet. 2003;362(9396):1659-66.

24. van Assen S, Agmon-Levin N, Elkayam O, et al. EULAR recommendations for vaccination in adult patients with autoimmune inflammatory rheumatic diseases. Ann Rheum Dis. 2011;70(3):414.

25. Maillefert JF, Sibilia J, Toussirot E, et al. Rheumatic disorders developed after hepatitis B vaccination. Rheumatology (Oxford). 1999;38(10):978-83.

26. Crowe SR, Merrill JT, Vista ES, et al. Influenza vaccination responses in human systemic lupus 
erythematosus: impact of clinical and demographic features. Arthritis Rheum. 2011;63(8):2396-406.

27. Petri M. Disease activity assessment in SLE: do we have the right instruments? Ann Rheum Dis. 2007;66(Suppl 3):iii61-4.

28. Hung JCH, Li KKW. Implications of COVID-19 for uveitis patients: perspectives from Hong Kong. Eye. 2020;34(7):1163-4.

29. Agarwal AK, Sudharshan S, Mahendradas $P$, et al. Impact of COVID-19 pandemic on uveitis patients receiving immunomodulatory and biological therapies (COPE STUDY). Br J Ophthalmol. 2020. https://doi.org/10.1136/bjophthalmol-2020317417.

30. Yuan P, Ai P, Liu Y, et al. Safety, tolerability, and immunogenicity of COVID-19 vaccines: a systematic review and meta-analysis. medRxiv. 2020. https://doi.org/10.1101/2020.11.03.20224998.

31. Castanha PMS, Nascimento EJM, Braga C, et al. Enhancement of Zika infection by dengue-specific antibodies does not alter the production of interleukin 6 in Fc $\gamma$ RII-expressing K562 cells. J Infect Dis. 2017;216(5):614-5.

32. Lurie N, Saville M, Hatchett R, Halton J. Developing Covid-19 vaccines at pandemic speed. N Engl J Med. 2020;382(21):1969-73.

33. Wang N, Shang J, Jiang S, Du L. Subunit vaccines against emerging pathogenic human coronaviruses. Front Microbiol. 2020;11:298-298.

34. Kaur SP, Gupta V. COVID-19 vaccine: a comprehensive status report. Virus Res. 2020;288: 198114-198114.

35. Wan Y, Shang J, Shihui S, et al. Molecular mechanism for antibody-dependent enhancement of coronavirus entry. J Virol. 2020;94(5):e02015e2019.

36. Su S, Du L, Jiang S. Learning from the past: development of safe and effective COVID-19 vaccines. Nat Rev Microbiol. 2021;19(3):211-219.

37. Wang H, Zhang Y, Huang B, et al. Development of an inactivated vaccine candidate, BBIBP-CorV, with potent protection against SARS-CoV-2. Cell. 2020;182(3):713-21.

38. Gao Q, Boa L, Mao H, et al. Development of an inactivated vaccine candidate for SARS-CoV-2. Science. 2020;369(6499):77.

39. Simonovich VA, Burgos Pratx LD, Scibona P, et al. A randomized trial of convalescent plasma in Covid-
19 severe pneumonia. N Engl J Med. 2021;384(7): 619-629.

40. Ouyang J, Isnard S, Lin J, et al. Convalescent plasma: the relay baton in the race for coronavirus disease 2019 treatment. Front Immunol. 2020;11: 2424.

41. Libster R, Marc GP, Wappner D, et al. Early hightiter plasma therapy to prevent severe Covid-19 in older adults. N Engl J Med. 2021;384(7):610-618.

42. Lee WS, Wheatley AK, Kent SJ, DeKosky BJ. Antibody-dependent enhancement and SARS-CoV-2 vaccines and therapies. Nat Microbiol. 2020;5(10): 1185-91.

43. Ju B, Zhang Q, Ge J, et al. Human neutralizing antibodies elicited by SARS-CoV-2 infection. Nature. 2020;584(7819):115-9.

44. Lv H, Wu NC, Tsang OTY, et al. Cross-reactive antibody response between SARS-CoV-2 and SARSCoV infections. Cell Rep. 2020;31(9): 107725-107725.

45. Sagar M, Reifler K, Rossi M, et al. Recent endemic coronavirus infection is associated with less severe COVID-19. J Clin Invest. 2020;131:1.

46. Aggarwal K, Agarwal A, Jaiswal N, et al. Ocular surface manifestations of coronavirus disease 2019 (COVID-19): a systematic review and meta-analysis. PLoS One. 2020;15(11):e0241661.

47. Güemes-Villahoz N, Burgos-Blasco B, Arribi-Vilela A, et al. Detecting SARS-CoV-2 RNA in conjunctival secretions: is it a valuable diagnostic method of COVID-19? J Med Virol. 2021;93(1):383-8.

48. Cheema M, Aghazadeh H, Nazarali S, et al. Keratoconjunctivitis as the initial medical presentation of the novel coronavirus disease 2019 (COVID-19). Can J Ophthalmol. 2020;55(4):e125-9.

49. Khavandi S, Tabibzadeh E, Naderan M, Shoar S. Corona virus disease-19 (COVID-19) presenting as conjunctivitis: atypically high-risk during a pandemic. Cont Lens Anterior Eye. 2020;43(3):211-2.

50. Nayak B, Poddar C, Panigrahi MK, Tripathy S, Mishra B. Late manifestation of follicular conjunctivitis in ventilated patient following COVID-19 positive severe pneumonia. Indian $\mathrm{J}$ Ophthalmol. 2020;68(8):1675-7.

51. Sindhuja K, Lomi N, Asif MI, Tandon R. Clinical profile and prevalence of conjunctivitis in mild COVID-19 patients in a tertiary care COVID-19 hospital: a retrospective cross-sectional study. Indian J Ophthalmol. 2020;68(8):1546-50. 
52. Badawi AE, Elsheikh SS, Addeen SZ, et al. An ophthalmic insight into novel coronavirus 2019 disease: a comprehensive review of the ocular manifestations and clinical hazards. J Curr Ophthalmol. 2020;32(4):315.

53. Wu P, Duan F, Luo C, et al. Characteristics of ocular findings of patients with coronavirus disease 2019 (COVID-19) in Hubei province, China. JAMA Ophthalmol. 2020;138(5):575-8.

54. Chen L, Deng C, Chen X, et al. Ocular manifestations and clinical characteristics of 535 cases of COVID-19 in Wuhan, China: a cross-sectional study. Acta Ophthalmol. 2020;98(8):e951-9.

55. Guo D, Xia J, Wang Y, Zhang X, Shen Y, Tong JP. Relapsing viral keratoconjunctivitis in COVID-19: a case report. Virol J. 2020;17(1):97.

56. Navel V, Chiambaretta F, Dutheil F. Haemorrhagic conjunctivitis with pseudomembranous related to SARS-CoV-2. Am J Ophthalmol Case Rep. 2020;2020:100735.

57. Méndez Mangana C, Barraquer Kargacin A, Barraquer RI. Episcleritis as an ocular manifestation in a patient with COVID-19. Acta Ophthalmol. 2020;98(8):e1056-7.

58. Bostanci Ceran B, Ozates S. Ocular manifestations of coronavirus disease 2019. Graefes Arch Clin Exp Ophthalmol. 2020;258(9):1959-63.
59. Marinho PM, Marcos AAA, Romano AC, Nascimento $\mathrm{H}$, Belfort R Jr. Retinal findings in patients with COVID-19. Lancet. 2020;395(10237):1610.

60. Insausti-García A, Reche-Sainz JA, Ruiz-Arranz C, Vázquez AL, Ferro-Osuna M. Papillophlebitis in a COVID-19 patient: inflammation and hypercoagulable state. Eur J Ophthalmol. 2020;2020: 1120672120947591-1120672120947591.

61. Casagrande M, Fitzek A, Püschel K, et al. Detection of SARS-CoV-2 in human retinal biopsies of deceased COVID-19 patients. Ocular Immunol Inflamm. 2020;28(5):721-5.

62. Dinkin M, Gao V, Kahan J, et al. COVID-19 presenting with ophthalmoparesis from cranial nerve palsy. Neurology. 2020;95(5):221.

63. Gutiérrez-Ortiz C, Méndez-Guerrero A, Rodrigo-Rey $\mathrm{S}$, et al. Miller Fisher syndrome and polyneuritis cranialis in COVID-19. Neurology. 2020;95(5):e601.

64. Belser JA, Rota PA, Tumpey TM. Ocular tropism of respiratory viruses. Microbiol Mol Biol Rev MMBR. 2013;77(1):144-56.

65. Zhang X, Chen X, Chen L, et al. The evidence of SARS-CoV-2 infection on ocular surface. Ocular Surf. 2020;18(3):360-2. 